\title{
Effect of Wnt5a on drug resistance in estrogen receptor-positive breast cancer
}

\author{
Ai Amioka ${ }^{1} \cdot$ Takayuki Kadoya $^{1}$ - Satoshi Sueoka ${ }^{1} \cdot$ Yoshie Kobayashi $^{1} \cdot$ Shinsuke Sasada $^{1} \cdot$ Akiko Emi $^{1}$. \\ Norio Masumoto ${ }^{1} \cdot{\text { Masaoki } \text { Ito }^{1} \cdot \text { Koh Nakayama }}^{2} \cdot$ Morihito Okada $^{1}$
}

Received: 30 October 2020 / Accepted: 13 March 2021 / Published online: 28 May 2021

(c) The Author(s) 2021

\begin{abstract}
Background Previously, we reported that Wnt5a-positive breast cancer can be classified as estrogen receptor (ER)-positive breast cancer; its prognosis is worse than that of Wnt5a-negative breast cancer. This study aimed to investigate the mechanisms underlying the poor prognosis in Wnt5a-positive breast cancer patients.

Methods In total, 151 consecutive ER-positive breast cancer patients who underwent resection between January 2011 and February 2014 were enrolled. DNA microarray and pathway analyses were conducted using MCF-7 cells stably expressing Wnt5a [MCF-7/Wnt5a (+)]. Based on the outcomes, cell viability/drug sensitivity assays, and mutation analysis were performed using cell cultures and breast cancer tissues. The relationship between Wnt5a and the PI3K-AKT-mTOR signaling pathway was also examined.

Results The relapse-free survival rate in patients with Wnt5a-positive breast cancer was significantly lower than that in patients with Wnt5a-negative breast cancer $(P=0.047)$. DNA microarray data suggest that only the cytochrome P450 (CYP) pathway was significantly upregulated in MCF-7/Wnt5a (+) cells $(P=0.0440)$. Additionally, MCF-7/Wnt5a (+) cells displayed reduced sensitivity to the metabolic substrates of CYP, tamoxifen $(P<0.001)$, paclitaxel $(P<0.001)$, and cyclophosphamide $(P<0.001)$. Of note, PIK3CA mutations were not associated with the expression of Wnt5a in breast cancer tissue and culture cells.

Conclusions In ER-positive breast cancer, Wnt5a upregulates the CYP metabolic pathway and suppresses tamoxifen, paclitaxel, and cyclophosphamide resistance, all of the three, standard treatment methods for ER-positive breast cancer. Wnt5a is thus potentially involved in the poor prognosis of ER-positive breast cancer independently of the PI3K-AKT-mTOR signaling pathway.
\end{abstract}

Keywords Wnt5a · Estrogen receptor-positive breast cancer $\cdot$ Cytochrome P450 $~$ Pathway analysis · Drug resistance

\section{Introduction}

The Wnt pathway is classified into the $\beta$-catenin-dependent and -independent pathways [1]. Wnt5a is a typical ligand of the $\beta$-catenin-independent pathway, modulating cell polarity and migration via the PCP/CE and $\mathrm{Ca}^{2+}$ pathways $[1,2]$. The

Takayuki Kadoya

takayukikadoya@gmail.com

1 Department of Surgical Oncology, Research Institute for Radiation Biology and Medicine, Hiroshima University, 1-2-3 Kasumi, Minami-Ku, Hiroshima 734-8551, Japan

2 Oxygen Biology Laboratory, Medical Research Institute, Tokyo Medical and Dental University, Bunkyo-ku, Tokyo 113-8510, Japan
Wnt5a signaling pathway is involved in the progression of several cancers [1, 3-7]; Wnt5a contributes to the progression of gastric, lung, and prostate cancers [3-5], but serves as a tumor suppressor in thyroid and ovarian cancers $[6,7]$. $\mathrm{Wnt5a}$ is reportedly overexpressed in approximately $30 \%$ of all breast cancer cases, and most Wnt5a-positive breast cancers are estrogen receptor (ER)-positive breast cancers. Of note, the 5-year relapse-free survival (RFS) rate is significantly lower in Wnt5a-positive versus -negative breast cancer [8].

Ovarian cancer cells overexpressing Wnt5a showed low chemosensitivity to paclitaxel and epirubicin [9]. However, in breast cancers, relatively few studies have examined the association between Wnt5a and drug sensitivity. Furthermore, upregulated PI3K-AKT-mTOR signaling is 
associated with a poor prognosis in ER-positive breast cancer [10-12]. Of note, Wnt5a is reportedly upregulated in ER-positive breast cancers harboring a PIK3CA mutation [13], indicating a close relationship between PI3K signaling and the Wnt5a pathway. Therefore, in the present study, the signaling pathways associated with Wnt5a were investigated to analyze the mechanisms underlying a Wnt5a-mediated drug resistance and poor prognosis.

\section{Materials and methods}

\section{Patients and breast cancer tissues (RFS-tracked cases)}

Consecutive breast cancer tissues resected between 2011 and 2014 were reviewed as previously described [8]. The followup period was extended, and the 8-year RFS was investigated by December 2019 in the present study. To strictly investigate "recurrence of early-stage breast cancer," the two patients with Stage IV at diagnosis included in our previous study [8], were excluded from this study.

Furthermore, we grouped patients treated with the key drugs, tamoxifen, paclitaxel, cyclophosphamide, epirubicin and 5-fluorouracil, and investigated RFS comparing Wnt5apositive and -negative patients.

\section{Cell culture}

The breast cancer cell lines MCF-7, and MDA-MB-175VII (RRID: CVCL_0031, and CVCL_1400) were obtained from ATCC (catalog \#HTB-22 and \#HTB-25, Manassas, VA, USA) and were confirmed not to be listed in the ICLAC Register of Misidentified Cell Lines (version 10). MDAMB-175-VII is an ER-positive and HER2-negative breast cancer cell line like MCF-7; however, unlike MCF-7, it endogenously expresses Wnt5a [8]. MCF-7 and MDA-MB175 -VII cells were cultured according to the manufacturer's instructions.

\section{Transfection, and RNA interference}

The pPGK-neo/Wnt5a plasmid was transfected into MCF-7 cells using Lipofectamine LTX + PLUS reagent (Life Technologies, Carlsbad, CA, USA). Successfully transfected cells selectively formed colonies in the presence of G418. The colonies were screened for Wnt5a expression through western blotting. Thereafter, certain MCF-7 cells stably expressing Wnt5a [MCF-7/Wnt5a (+)] or not expressing Wnt5a [MCF-7/Wnt5a (-)] were established. Additionally, the siRNA-mediated suppression of Wnt5a in MCF-7 and MDA-MB-175-VII cells was conducted as previously described [8].

\section{Gene microarray analyses}

The Oligo DNA microarray analyses were performed using 3D-Gene Human Oligo chip $25 \mathrm{k}$ (Toray Industries, Tokyo, Japan) as previously described [8]. In all three MCF-7/Wnt5a (+) clones, only significantly upregulated (expression $\geq$ twofold) or downregulated (expression $\leq 1 / 2$ ) genes were selected. Gene ontology and pathway analyses were performed using the DAVID online tool (Version 6.8, https://david.ncifcrf.gov/). In addition, a heatmap was generated using SHINYHEATMAP.COM (http://shinyheatm ap.com/). Among the differentially expressed genes, we searched for those associated with breast cancer/cancer/ CYP, as per the PubMed website (https://pubmed.ncbi. nlm.nih.gov/). The genes and interrelationships were then arranged using the application "GeneMania" in Cytoscape (Version 3.8.2, http://cytoscape.org).

\section{Cell viability assay}

$6 \times 10^{3}$ MCF-7/Wnt5a (+) and MCF-7/Wnt5a (-) cells from $\geq 80 \%$ confluent cultures were seeded into a 96 -well plate in triplicate. Twenty-four, 48 , and $72 \mathrm{~h}$ after the administration of $15 \mu \mathrm{M}$ tamoxifen, $200 \mathrm{nM}$ paclitaxel, $8000 \mu \mathrm{M}$ cyclophosphamide, $800 \mathrm{nM}$ epirubicin, and $400 \mu \mathrm{M}$ 5-fluorouracil, $20 \mu \mathrm{L}$ CellTiter $96^{\circledR}$ Aqueous One Solution Reagent (Promega, Madison, WI, USA) was added into each well (please see Online Resource 1A). Two hours later, the absorbance was measured according to the manufacture's instruction. Additionally, viability was also measured in the context of MDA-MB-175-VII cells. Briefly, $24 \mathrm{~h}$ after seeding (in the same way as for MCF-7 cells) MDA-MB-175-VII were transfected with the Wnt5a-siRNA or negative-siRNA (catalog \#4392420 or \#4390843, Life Technologies) and viability was measured as above, 24,48 , and $72 \mathrm{~h}$ later.

\section{Western blot analysis}

For immunoblot analysis, MCF-7 and MDA-MB-175-VII cells were washed with PBS and lysed with lysis buffer containing a Phosphatase Inhibitor Cocktail (Nacalai Tesque Inc., Kyoto, Japan). Proteins were separated via SDS-PAGE and then electro-transferred onto nitrocellulose membranes (Amersham Protran Premium, GE Healthcare, Buckinghamshire, UK). The membranes were probed with various primary and secondary antibodies (Online Resource 1B) and visualized with enhanced chemiluminescence detection reagents (Amersham ECL Select, GE Healthcare, Buckinghamshire, UK). All western blotting experiments were performed in triplicate. 


\section{Detection of PIK3CA mutant variants}

Among the 151 cases immunoreactive for Wnt5a, PIK3CA mutations were evaluated only in those with a tumor size of $\geq 1 \mathrm{~cm}$ in diameter. The QIAamp DNA FFPE Tissue Kit (Qiagen GmbH, Hilden, Germany) was used to extract DNA from formalin-fixed paraffin-embedded (FFPE) tissues. The E542K, E545D/K, and H1047R/L were detected through direct sequencing using the primers listed in Online Resource 1C.

\section{Quantification of Wnt5a mRNA expression}

RNA was extracted using the NucleoSpin total RNA FFPE (Takara Bio, Shiga, Japan) from tissues sections sliced from the FFPE block, including the tumor component only. cDNA was synthesized through reverse-transcription using the PrimeScript II High Fidelity RT-PCR Kit (Takara Bio). Wnt5a expression was quantitatively analyzed via real-time PCR using the SsoFast EvaGreen Supermix (Bio-Rad, Hercules, CA, USA) and the CFX96 real-time PCR detecting system (Bio-Rad). Wnt5a expression was quantified using the $\Delta \mathrm{Ct}$ value. The used primers are listed in Online Resource 1C.

\section{Statistical analysis}

Statistical analysis was performed using the EZR [14] and SPSS (Version 20.0, Chicago, IL, USA) software. Welch's $t$ test was used to compare the age, and cell viability between Wnt5a-negative and -positive cells, and Wnt5a-silenced and -non-silenced cells. The clinicopathologic characteristics were analyzed using the Chi-squared test. The significance between RFS curves was analyzed using the generalized Wilcoxon test. The frequency of Wnt5a positivity and the expression levels of Wnt5a mRNA were compared between $P I K 3 C A$ mutation-negative and -positive cases using the Chi-square test and Welch's $t$-test, respectively. $P$ values $<$ 0.05 were considered statistically significant.

\section{Results}

\section{Wnt5a-positive breast cancer patients show poor prognosis}

A total of 151 patients was enrolled. The median (range) follow-up period was 73.2 (11.7 to 102) months for all patients. The background of the patients is shown in Online Resource 2. The 8-year RFS rate of Wnt5a-positive breast cancer patients was lower than that of Wnt5a-negative patients $[(91.9 \%(95 \% \mathrm{CI}=85.1-98.7)$ vs $98.6 \%,(95 \%$

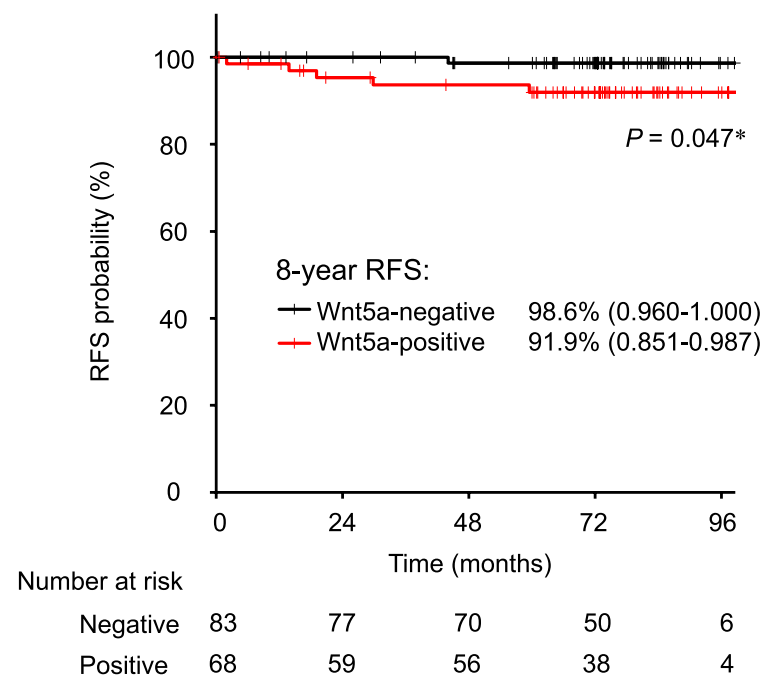

Fig. 1 Prognosis of Wnt5a in ER-positive breast cancer patients. Prognosis was estimated via Kaplan-Meier analysis $(n=151)$; Wnt5a-positive breast cancer patients $(n=68)$ displayed a lower 8-year RFS probability: $* P=0.047$ (Wilcoxon test). $R F S$ relapse-free survival

$\mathrm{CI}=96.0-100.0$ ), $P=0.047$ ] (Fig. 1). The postoperative treatment regimens used in recurrent patients are listed in Online Resource 3.

\section{The CYP pathway is upregulated in Wnt5a-expressing MCF-7 cells}

To investigate pathways related to the recurrence of Wnt5apositive breast cancer, MCF-7 cells stably expressing Wnt5a were established and DNA microarray analyses were performed. A total of 176 candidate genes differentially expressed between MCF-7/Wnt5a (+) and MCF-7/Wnt5a $(-)$ cells, were identified; 76 unique genes were upregulated (expression $\geq$ twofold) and 100 unique genes were downregulated (expression $\leq 1 /$ twofold; Online Resource $4)$. The $P$-value was determined using the DAVID database (Table 1). Interestingly, as per the Kyoto Encyclopedia of Genes and Genomes (KEGG) pathway analysis, physiological pathways directly or indirectly influencing Wnt5a expression were identified; only the cytochrome P450 (CYP) pathway, involved in drug metabolism [15], was identified among the upregulated genes $(P=0.0440$; Table 1). Conversely, certain virus-related pathways were identified among the downregulated genes (Table 1). Interestingly, the JAK-STAT signaling pathway, associated with cancer progression, was attenuated in MCF-7/Wnt5a (+) cells (Table 1). Of note, the upregulated genes were primarily involved in the oxidation-reduction process, response to calcium ions, retinoid metabolic process, response to 
Table 1 Selected gene categories significantly overrepresented in Wnt5a-positive breast cancer

\begin{tabular}{|c|c|c|c|}
\hline Gene category & Number of genes & $\%$ & $P$-value \\
\hline \multicolumn{4}{|l|}{ Upregulated genes } \\
\hline \multicolumn{4}{|l|}{ KEGG pathway } \\
\hline Drug metabolism—cytochrome P450 & 3 & 4.2 & 0.044 \\
\hline Chemical carcinogenesis & 3 & 4.2 & 0.059 \\
\hline \multicolumn{4}{|l|}{ Gene ontology } \\
\hline \multicolumn{4}{|l|}{ Biological process } \\
\hline Oxidation-reduction process & 8 & 11 & 0.006 \\
\hline Response to calcium ion & 3 & 4.2 & 0.019 \\
\hline Retinoid metabolic process & 3 & 4.2 & 0.021 \\
\hline Response to hypoxia & 4 & 5.6 & 0.025 \\
\hline Retinal metabolic process & 2 & 2.8 & 0.043 \\
\hline Protein polymerization & 2 & 2.8 & 0.046 \\
\hline \multicolumn{4}{|l|}{ Cellular component } \\
\hline Extracellular exosome & 20 & 28 & 0.003 \\
\hline Early endosome & 5 & 7 & 0.009 \\
\hline Plasma membrane & 23 & 32 & 0.021 \\
\hline Endosome membrane & 4 & 5.6 & 0.027 \\
\hline \multicolumn{4}{|l|}{ Molecular function } \\
\hline ATPase binding & 3 & 4.2 & 0.028 \\
\hline Oxidoreductase activity & 4 & 5.6 & 0.034 \\
\hline \multicolumn{4}{|l|}{ Downregulated genes } \\
\hline \multicolumn{4}{|l|}{ KEGG pathway } \\
\hline Influenza A & 13 & 13.8 & $<0.001$ \\
\hline Herpes simplex infection & 13 & 13.8 & $<0.001$ \\
\hline Measles & 11 & 11.7 & $<0.001$ \\
\hline Hepatitis C & 9 & 9.6 & $<0.001$ \\
\hline Hepatitis B & 6 & 6.4 & 0.002 \\
\hline Toxoplasmosis & 5 & 5.3 & 0.004 \\
\hline Epstein-Barr virus infection & 5 & 5.3 & 0.006 \\
\hline RIG-I-like receptor signaling pathway & 4 & 4.3 & 0.008 \\
\hline Chemokine signaling pathway & 5 & 5.3 & 0.024 \\
\hline JAK-STAT signaling pathway & 4 & 4.3 & 0.055 \\
\hline Cytosolic DNA-sensing pathway & 3 & 3.2 & 0.055 \\
\hline \multicolumn{4}{|l|}{ Gene ontology } \\
\hline \multicolumn{4}{|l|}{ Biological Process } \\
\hline Type I interferon signaling pathway & 19 & 20.2 & $<0.001$ \\
\hline Defense response to virus & 22 & 23.4 & $<0.001$ \\
\hline Response to virus & 19 & 20.2 & $<0.001$ \\
\hline Negative regulation of viral genome replication & 13 & 13.8 & $<0.001$ \\
\hline Interferon-gamma-mediated signaling pathway & 10 & 10.6 & $<0.001$ \\
\hline Response to interferon-alpha & 5 & 5.3 & $<0.001$ \\
\hline Innate immune response & 12 & 12.8 & $<0.001$ \\
\hline Response to interferon-beta & 4 & 4.3 & $<0.001$ \\
\hline Negative regulation of type I interferon production & 4 & 4.3 & $<0.001$ \\
\hline Cellular response to interferon-alpha & 3 & 3.2 & $<0.001$ \\
\hline Positive regulation of interferon-alpha production & 3 & 3.2 & 0.0015 \\
\hline Positive regulation of interferon-beta production & 3 & 3.2 & 0.0076 \\
\hline \multicolumn{4}{|l|}{ Cellular component } \\
\hline Cytosol & 37 & 39.4 & $<0.001$ \\
\hline Cytoplasm & 37 & 39.4 & 0.0073 \\
\hline Plasma membrane & 31 & 33 & 0.0076 \\
\hline
\end{tabular}


Table 1 (continued)

\begin{tabular}{lccr}
\hline Gene category & Number of genes & P & -value \\
\hline Spermatoproteasome complex & 2 & 2.1 & 0.024 \\
Molecular function & & & \\
Double-stranded RNA binding & 8 & 8.5 & $<0.001$ \\
2'-5'-oligoadenylate synthetase activity & 4 & 4.3 & $<0.001$ \\
NAD+ ADP - ribosyltransferase activity & 4 & 4.3 & $<0.001$ \\
Single-stranded RNA binding & 4 & 4.3 & 0.001 \\
Nucleotidyltransferase activity & 3 & 3.2 & 0.0067 \\
Helicase activity & 4 & 4.3 & 0.0069 \\
Transferase activity & 4 & 4.3 & 0.0096 \\
CCR5 chemokine receptor binding & 2 & 2.1 & 0.036 \\
\hline
\end{tabular}

hypoxia and retinal metabolic process, ATPase binding, and oxidoreductase activity (Table 1). Conversely, most of the downregulated genes include those related to viruses and interferons (Table 1).

\section{Relationships between differentially expressed genes, cancer, and CYP}

The 176 differentially expressed genes are represented in the form of a heatmap (Fig. 2). Among them, 21 genes are related to breast cancer/cancer/CYP and have been reported to promote cell migration and proliferation, and cancer metastasis. The upregulated genes that correlate positively with the malignancy of ER-positive breast cancer are UGT2B15 [16], AKRIC3 [17], ALCAM [8], and PTPRN2 [18]; the downregulated genes are JAK2, STAT1 [19], CCNA1 [20]. Of note, UGT2B15 and AKRIC3 are genes related to the CYP pathway, and $A L C A M$ and PTPRN2 are genes co-expressed with CCNA1 (Online Resource 5).

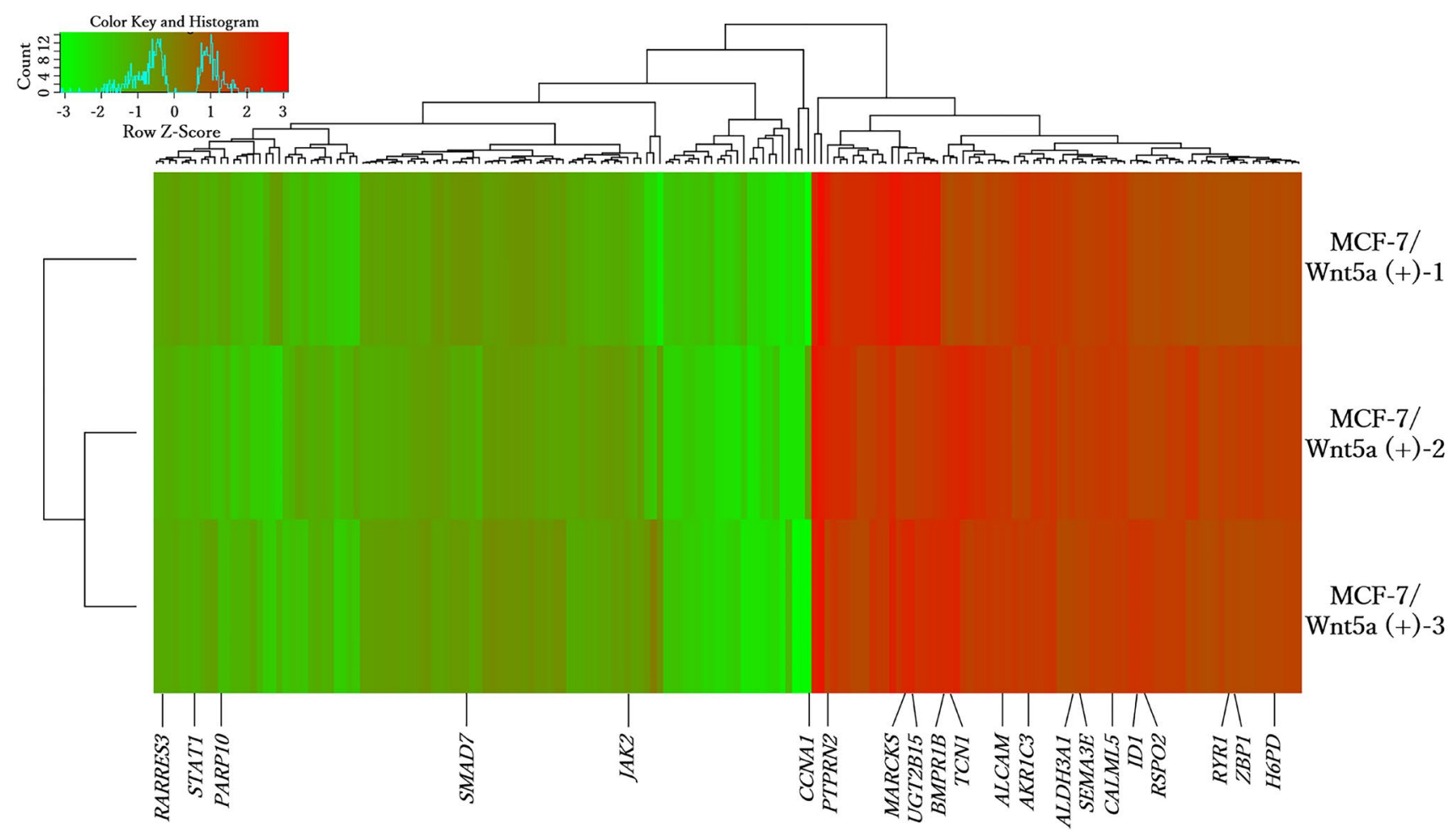

Fig. 2 Gene expression heatmap. Gene expression was analyzed via microarray using three MCF-7/Wnt5a (+) clones (MCF-7/Wnt5a (+) 1 to 3; see Online Resource 4). Gene expression was quantified as the relative ratio based on control cells [MCF-7/Wnt5a (-)]. The heatmap was generated at http://shinyheatmap.com/ after the expression levels were logarithmized 


\section{Wnt5a expression decreases the sensitivity to tamoxifen, paclitaxel, and cyclophosphamide}

Tamoxifen, paclitaxel and cyclophosphamide, but not epirubicin and 5-fluorouracil, are metabolized by CYP [21-25]. Therefore, the sensitivity to these five drugs, used in the standard treatment of ER-positive breast cancer, was examined. MCF-7/Wnt5a (+) cells exhibited significantly lower sensitivity to tamoxifen, paclitaxel, and cyclophosphamide than MCF-7/Wnt5a (-) cells at both $48 \mathrm{~h}$ and $72 \mathrm{~h}(P<0.001 ;$ Fig. 3a-c). In contrast, no difference was found in resistance to epirubicin $(P=0.12)$ and MCF-7/ a

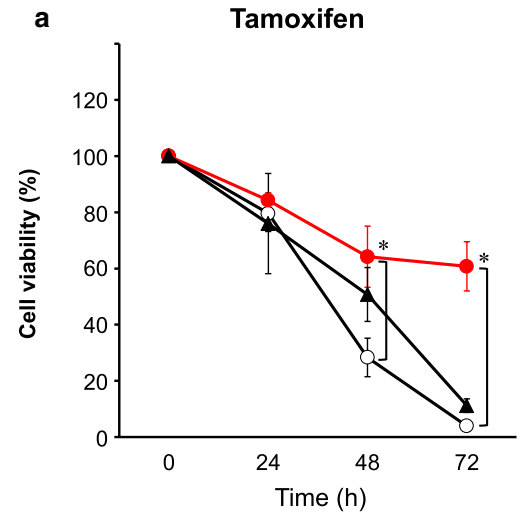

$* P<0.001$

C

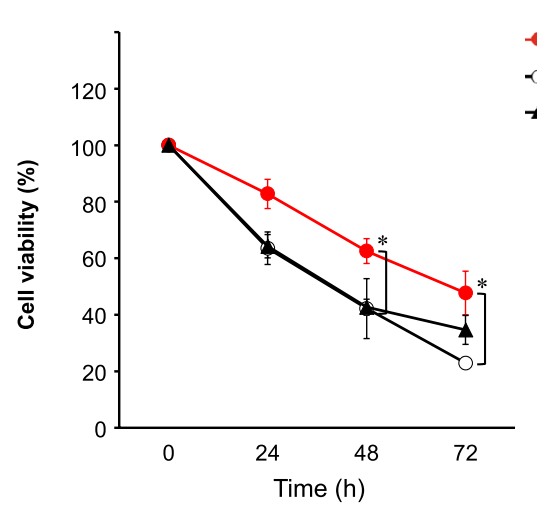

$* P<0.001$ $\rightarrow$ MCF-7/Wnt5a (+)

$\sim$ MCF-7/Wnt5a (-)

$\leftarrow$ MCF-7 normal b

نे

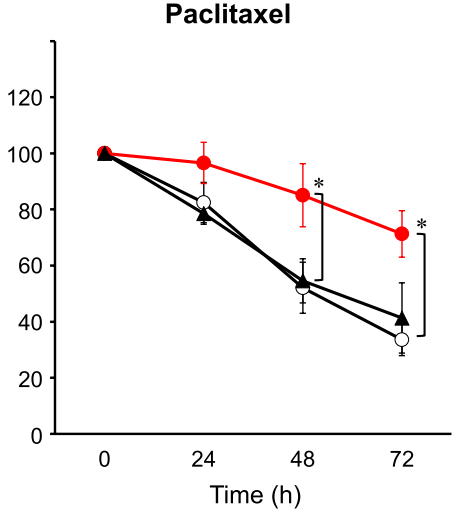

$* P<0.001$

d

- MCF-7/Wnt5a (+)

MCF-7/Wnt5a (-)

^ MCF-7 normal

نั

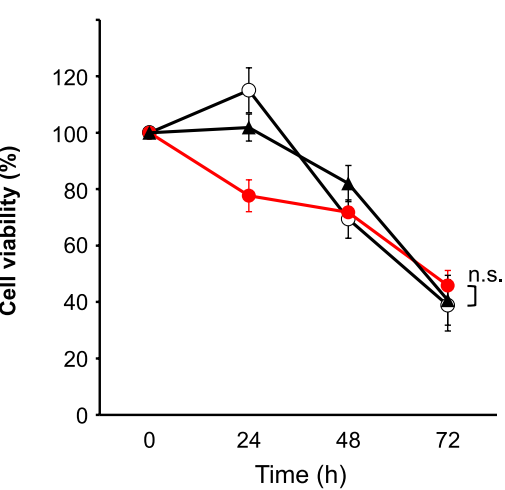

- MCF-7/Wnt5a (+)

$\rightarrow$ MCF-7/Wnt5a (-)

$\rightarrow$ MCF-7 normal
Fig. 3 Wnt5a expression diminishes the sensitivity to tamoxifen, paclitaxel, and cyclophosphamide. MCF-7, MCF-7/Wnt5a (+), and MCF-7/Wnt5a (-) cells were exposed to $15 \mu \mathrm{M}$ tamoxifen (a), $200 \mathrm{nM}$ paclitaxel (b), $8000 \mu \mathrm{M}$ cyclophosphamide (c), $800 \mathrm{nM}$ epirubicin (d), or $400 \mu \mathrm{M} 5$-fluorouracil (e). Results are represented as the mean \pm S.D. of 6 measurements. $* P<0.001 ; * * P=0.021$ (Welch's $t$-test); n.s. not significant 
Wnt5a (+) cells exhibited higher sensitivity to 5-fluorouracil ( $P=0.021$; Fig. 3d, e). Importantly, similar trends were observed in the context of MDA-MB-175-VII cells, although without significance in some instances (Online Resource 6).

Additionally, we examined the difference in the RFS between Wnt5a-positive versus -negative breast cancer patients treated with the different above drugs. The RFS probability was lower in the Wnt5a-positive. However, used. However, no significant differences were detected, probably due to the small sample-size (Online Resource 7).

\section{The PI3K signaling pathway is not correlated with Wnt5a expression}

Next, the relationship between the expression of Wnt5a and the PI3K and JNK signaling pathways was examined via western blotting in MCF-7/Wnt5a (+) and MCF-7/Wnt5a (-) cells. The expression of phosphorylated JNK, which occurs downstream of the Wnt5a signaling pathway [2], remained unaltered in Wnt5a overexpressing or silenced

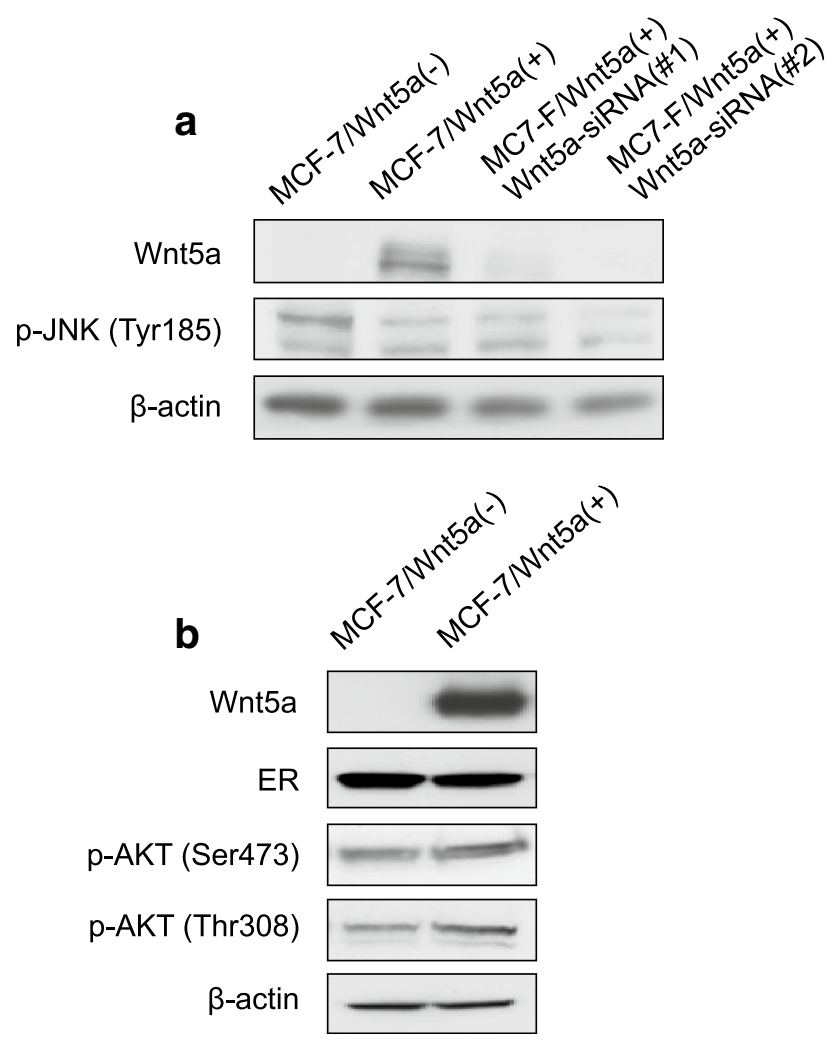

Fig. 4 Effect of Wnt5a on the expression of breast cancer-related signaling molecules. The expression of phosphorylated JNK (a) and of phosphorylated AKT (b) was assessed via western blotting. ER estrogen receptor cells (Fig. 4a). Similarly, there was no difference in the expression of phosphorylated AKT (Fig. 4b).

PIK3CA mutations were examined in 40 cases (Table 2) and detected in 19 cases of ER-positive breast cancers (Table 3); three principal mutation sites were identified: E542K, E545K, and H1047R [26] (Fig. 5a). Of note, PIK3CA mutations were observed in 8 and $11 \mathrm{Wnt5a-posi-}$ tive and -negative breast cancer patients, respectively. However, there was no significant difference in the frequency of PIK3CA mutations depending on the expression of Wnt5a $(P=0.73$; Table 3$)$. Furthermore, no difference in Wnt5a expression was observed depending on the mutation site (Table 4).

Furthermore, the expression of Wnt5a mRNA. The median (range) expression of Wnt5a mRNA was 1.7 (0.94 to 3.9) in PIK3CA mutation-negative and $2.5(0.83-5.1)$ in PIK3CA mutation-positive cases; however, no significant difference was observed between the two groups $(P=0.92$; Fig. 5b).

Table 2 Characteristics of the 40 ER-positive breast cancer patients assessed for the PIK3CA status

\begin{tabular}{ll}
\hline & Total $(n=40)$ \\
\hline Age (median, range) & $58.5(87-34)$ \\
$\leq 50$ & 15 \\
$>50$ & 25 \\
Tumor size & \\
pT1 $20 \mathrm{~mm}$ & 17 \\
pT2/pT3 $>20$ mm & 23 \\
Lymph-node metastasis & \\
Negative & 25 \\
Positive & 15 \\
Progesterone receptor & \\
Negative & 2 \\
Positive & 38 \\
HER2 status & \\
Negative & 37 \\
Positive & 3 \\
Nuclear grade & \\
1, 2 & 13 \\
3 & 27 \\
Wnt5a expression (IHC) & \\
Wnt5a-negative & 21 \\
Wnt5a-positive & \\
\hline
\end{tabular}

IHC immunohistochemistry, HER2 human epidermal growth factor receptor 2 
Table 3 Wnt5a expression, assessed through immunohistochemistry (IHC), according to the PIK3CA mutation status

\begin{tabular}{llll}
\hline Total $(n=40)$ & \multicolumn{2}{l}{$P I K 3 C A$ mutation } & $P$-value \\
\cline { 2 - 3 } & $\begin{array}{l}\text { Negative } \\
(n=21)\end{array}$ & $\begin{array}{l}\text { Positive } \\
(n=19)\end{array}$ & \\
\hline Wnt5a expression (IHC) & & 11 & \\
Wnt5a-negative & 10 & 8 & 0.73 \\
Wnt5a-positive & 11 & & \\
\hline
\end{tabular}

\section{Discussion}

The recurrence rate of Wnt5a-positive breast cancer patients is significantly higher than that of Wnt5a-negative breast cancer patients. Therefore, this study investigated the association between the expression of Wnt5a expression and malignancy grade and prognosis. Interestingly, pathway analysis revealed that the CYP metabolic pathway was upregulated after Wnt5a overexpression.

CYP is a key enzyme that oxidizes various substrates and primarily metabolizes drugs in the liver. In our study, CYP upregulation reduced the sensitivity to tamoxifen, paclitaxel, and cyclophosphamide (all metabolized by CYP). Conversely, the sensitivity to epirubicin and 5-fluorouracil (not metabolized by CYP) was not affected. These results suggest that Wnt5a enhances the tamoxifen, paclitaxel, and cyclophosphamide metabolism via CYP, thus
Table 4 PIK3CA mutation sites in ER-positive breast cancers patients $(n=19)$, as detected through the Sanger method

\begin{tabular}{lllll}
\hline Wnt5a expression (IHC) & \multicolumn{2}{l}{$P I K 3 C A$ mutation } & $P$-value \\
\cline { 2 - 4 } & Exon 9 & Exon 20 & \\
\cline { 2 - 4 } & E542K & E545K & H1047R & \\
\hline Wnt5a-negative & 0 & 6 & 4 & \\
Wnt5a-positive & 1 & 3 & 5 & 0.50 \\
\hline
\end{tabular}

IHC immunohistochemistry

decreasing their intracellular concentration. Therefore, the early termination of adjuvant drug therapy for breast cancer may lead to a high recurrence rate of Wnt5a-positive breast cancer. Importantly, as no prior study have shown the association between Wnt5a and CYP in any cancer, these results are novel.

The genes associated in this study with Wnt5a in ERpositive breast cancer are summarized in Fig. 6 together with the previously reported genes [27-30]. Among the upregulated genes, UGT2B15 and AKRIC3 are included in the CYP metabolic pathway as per KEGG pathway analyses, suggesting the association between the CYP and Wnt5a pathways in the context of drug sensitivity [16, 17]. Interestingly, $A L C A M$ and PTPRN2 were also shown to correlate with Wnt5a expression and to promote cell migration $[8,18]$.
Fig. 5 a Occurrence of mutations within exons 9 and 20 of PIK3CA. b Wnt5a mRNA expression according to the PIK3CA mutation status $(n=40)$. The Wnt5a mRNA expression was examined in PIK3CA mutation-positive and -negative cancers. Statistical significance is highlighted: $* P=0.92$ (Welch's $t$-test)

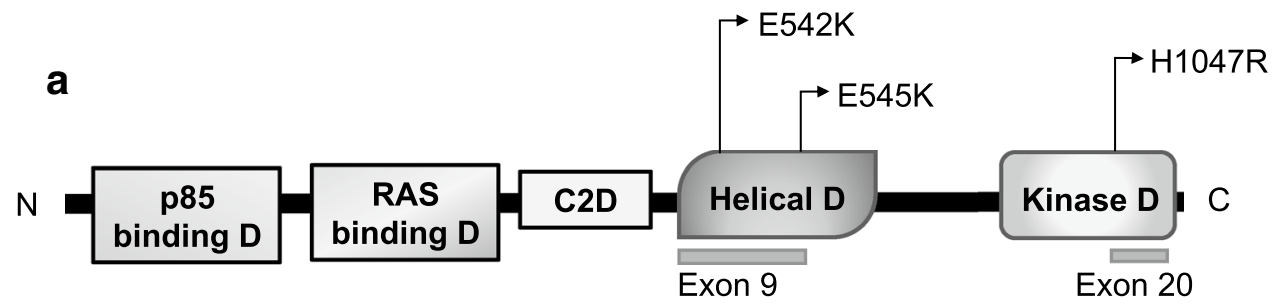

b

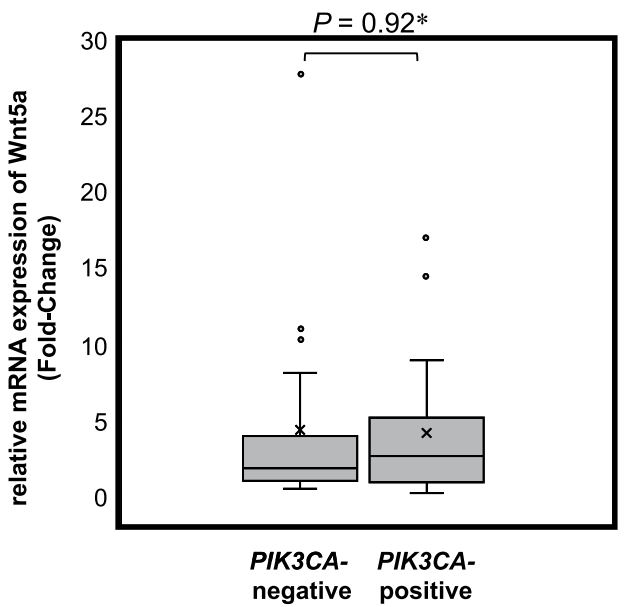


Fig. 6 The genes associated with Wnt5a in ER-positive breast cancer are summarized. The solid black lines represent the relationships already reported in the literature; the colored solid lines and the dotted lines and yellow line represent the relationships defined in this study. TAM tamoxifen, $P T X$ paclitaxel, CPA cyclophosphamide

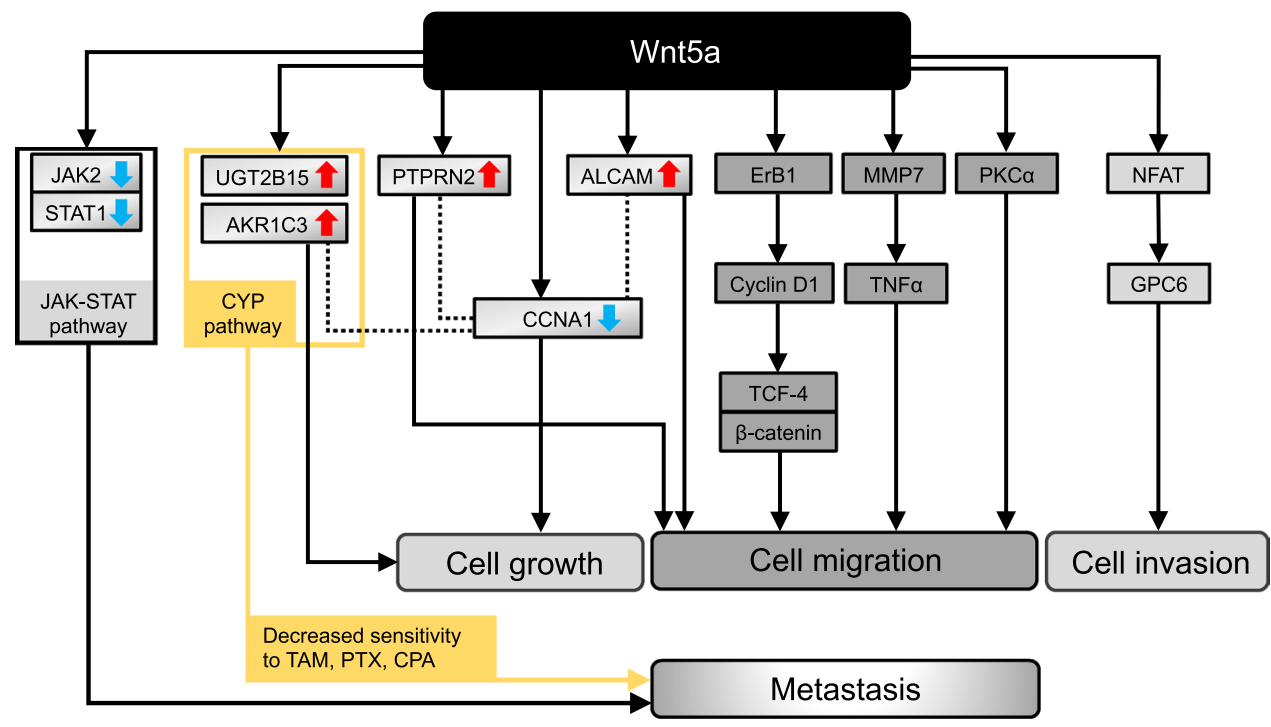

Additionally, the downregulation of the JAK-STAT signaling pathway was reported to contribute to breast cancer progression and metastasis [19]. Of note, it has been reported that the downregulation of $C C N A 1$ leads to cell growth in the context of breast cancer [20]. Interestingly, here we determined that $C C N A 1$ is co-expressed with ALCAM, PTPRN2, and AKRIC3, suggesting an association between them.

Based on our previous reports [12, 31, 32] we hypothesized that Wnt5a expression would be associated with the PI3K-AKT-mTOR signaling pathway. However, unexpectedly, no significant correlation was found. These results indicate the involvement of pathways other than PI3K in the recurrence of Wnt5a-positive breast cancer.

Altogether, our results indicate that Wnt5a potentially serves as a drug resistance marker in ER-positive breast cancer. However, further studies, in vivo, are essential to validate our results. Additionally, further studies are required for the development of new drugs targeting drug resistance markers; for instance, the development of a treatment strategy for Wnt5a-positive breast cancer based on anti-Wnt5a antibodies, in line with that developed for Wnt5a-positive gastric cancer [33] should be carried out.

In conclusion, here, we show that Wnt5a upregulates CYP expression and diminishes the sensitivity to key drugs used for treating ER-positive breast cancer, including tamoxifen, paclitaxel, and cyclophosphamide. Wnt5a is potentially involved in the poor prognosis of ER-positive breast cancer independently of the PI3K-AKT-mTOR signaling pathway.

Supplementary Information The online version contains supplementary material available at https://doi.org/10.1007/s12282-021-01241-0.
Acknowledgements We thank Yusuke Motoi for his assistance with the experiments. We also thank Editage (www.editage.com) for English language editing. This study was funded by a Grant-in-Aid for Young Scientists B (Grant number: 17K16509) and a Grant-in-Aid for Scientific Research C (Grant number: 17K10549) from the Ministry of Education, Culture, Sports, Science and Technology, Japan. This study was also supported in part by Nanken-Kyoten, TMDU (2020-A25 to T.K.). The funding sources had no role in study design.

Author contributions All authors contributed to the study conception and design. Material preparation, data collection, and analysis were performed by AA, TK, and MI. The first draft of the manuscript was written by AA and all authors commented on the previous versions of the manuscript. All authors read and approved the final version of the manuscript.

\section{Declarations}

Conflict of interest The authors have no competing interests to declare.

Ethical approval The study was approved by the institutional review board of Hiroshima University Hospital (No. 926) and was performed in accordance with the 1964 Helsinki Declaration and its later amendments or comparable ethical standards.

Informed consent Informed consent was obtained from all participants included in the study.

Open Access This article is licensed under a Creative Commons Attribution 4.0 International License, which permits use, sharing, adaptation, distribution and reproduction in any medium or format, as long as you give appropriate credit to the original author(s) and the source, provide a link to the Creative Commons licence, and indicate if changes were made. The images or other third party material in this article are included in the article's Creative Commons licence, unless indicated otherwise in a credit line to the material. If material is not included in the article's Creative Commons licence and your intended use is not permitted by statutory regulation or exceeds the permitted use, you will need to obtain permission directly from the copyright holder. To view a copy of this licence, visit http://creativecommons.org/licenses/by/4.0/. 


\section{References}

1. Kikuchi A, Yamamoto H, Sato A. Selective activation mechanisms of Wnt signaling pathways. Trends Cell Biol. 2009;19:119-29.

2. Cao Y, Wang X, Xu C, Gao Z, Zhou H, Wang Y, et al. 4-HPR impairs bladder cancer cell migration and invasion by interfering with the Wnt5a/JNK and Wnt5a/MMP-2 signaling pathways. Oncol Lett. 2016;12:1833-9.

3. Kurayoshi M, Oue N, Yamamoto H, Kishida M, Inoue A, Asahara $\mathrm{T}$, et al. Expression of Wnt-5a is correlated with aggressiveness of gastric cancer by stimulating cell migration and proliferation. Cancer Res. 2006;66:10439-48.

4. Huang C-L, Liu D, Nakano J, Ishikawa S, Kontan K, Yokomise H, et al. Wnt5a expression is associated with the tumor proliferation and the stromal vascular endothelial growth factor: an expression in non-small-cell lung cancer. J Clin Oncol. 2005;23:8765-73.

5. Yamamoto H, Oue N, Sato A, Hasegawa Y, Yamamoto H, Matsubara A, et al. Wnt5a signaling is involved in the aggressiveness of prostate cancer and expression of metalloproteinase. Oncogene. 2010;29:2036-46.

6. Kremenevskaja N, von Wasielewski R, Rao AS, Schöfl C, Andersson T, Braban G. Wnt-5a has tumor suppressor activity in thyroid carcinoma. Oncogene. 2005;24:2144-54.

7. Bitler BG, Nicodemus JP, Li H, Cai Q, Wu H, Hua X, et al. Wnt5a suppresses epithelial ovarian cancer by promoting cellular senescence. Cancer Res. 2011;71:6184-94.

8. Kobayashi Y, Kadoya T, Amioka A, Hanaki H, Sasada S, Masumoto N, et al. Wnt5a-induced cell migration is associated with the aggressiveness of estrogen receptor-positive breast cancer. Oncotarget. 2018;9:20979-92.

9. Peng C, Zhang X, Yu H, Wu D, Zheng J. Wnt5a as a predictor in poor clinical outcome of patients and a mediator in chemoresistance of ovarian cancer. Int J Gynecol Cancer. 2011;21:280-8.

10. Miller TW, Hennessy BT, González-Angulo AM, Fox EM, Mills GB, Chen H, et al. Hyperactivation of phosphatidylinositol-3 kinase promotes escape from hormone dependence in estrogen receptor-positive human breast cancer. J Clin Invest. 2010;120:2406-13.

11. Yang SX, Polley E, Lipkowitz S. New insights on PI3K/AKT pathway alterations and clinical outcomes in breast cancer. Cancer Treat Rev. 2016;45:87-96.

12. Steelman LS, Navolanic P, Chappell WH, Abrams SL, Wong EWT, Martelli AM, et al. Involvement of Akt and mTOR in chemotherapeutic- and hormonal-based drug resistance and response to radiation in breast cancer cells. Cell Cycle. 2011;10:3003-15.

13. Cizkova M, Cizeron-Clairac G, Vacher S, Susini A, Andrieu $\mathrm{C}$, Lidereau R, et al. Gene expression profiling reveals new aspects of PIK3CA mutation in ER alpha-positive breast cancer: Major implication of the Wnt signaling pathway. PLoS ONE. 2010;5:e15647.

14. Kanda Y. Investigation of the freely available easy-to-use software 'EZR' for medical statistics. Bone Marrow Transplant. 2013;48:452-8.

15. Yasuda K, Ranade A, Venkataramanan R, Strom S, Chupka J, Ekins S, et al. A comprehensive in vitro and in silico analysis of antibiotics that activate pregnane $\mathrm{X}$ receptor and induce CYP3A4 in liver and intestine. Drug Metab Dispos. 2008;36:1689-97.

16. Nowell SA, Ahn J, Rae JM, Scheys JO, Trovato A, Sweeney C, et al. Association of genetic variation in tamoxifen-metabolizing enzymes with overall survival and recurrence of disease in breast cancer patients. Breast Cancer Res Treat. 2005;91:249-58.
17. Jia Y, Domenico J, Swasey C, Wang M, Gelfand EW, Lucas JJ. Modulated expression of genes encoding estrogen metabolizing enzymes by G1-phase cyclin-dependent kinases 6 and 4 in human breast cancer cells. PLoS ONE. 2004;9:e97448.

18. Sengelaub CA, Navrazhina K, Ross JB, Halberg N, Tavazoie SF. PTPRN2 and PLC $\beta 1$ promote metastatic breast cancer cell migration through $\mathrm{PI}(4,5) \mathrm{P} 2$-dependent actin remodeling. EMBO J. 2016;35:62-76.

19. Yates LR, Knappskog S, Wedge D, Farmery JHR, Gonzalez S, Martincorena I, et al. Genomic evolution of breast cancer metastasis and relapse. Cancer Cell. 2017;32(169-184):e7.

20. Starlard-Davenport A, Kutanzi K, Tryndyak V, Word B, Lyn-Cook B. Restoration of the methylation status of hypermethylated gene promoters by microRNA-29b in human breast cancer: a novel epigenetic therapeutic approach. J Carcinog. 2013;12:15.

21. Coller JK, Krebsfaenger N, Klein K, Endrizzi K, Wolbold R, Lang $\mathrm{T}$, et al. The influence of $C Y P 2 B 6, C Y P 2 C 9$ and $C Y P 2 D 6$ genotypes on the formation of the potent antioestrogen Z-4-hydroxytamoxifen in human liver. Br J Clin Pharmacol. 2002;54:157-67.

22. Sonnichsen DS, Liu Q, Schuetz EG, Schuetz JD, Pappo M, Relling MV, et al. Variability in human cytochrome $\mathrm{P} 450$ paclitaxel metabolism. J Pharmacol Exp Ther. 1995;275:566-75.

23. Chang TK, Weber GF, Crespi CL, Waxman DJ. Differential activation of cyclophosphamide and ifosphamide by cytochromes $\mathrm{P}-4502 \mathrm{~B}$ and $3 \mathrm{~A}$ in human liver microsomes. Cancer Res. 1993;53:5629-37.

24. Robert J. Clinical pharmacokinetics of epirubicin. Clin Pharmacokinet. 1994;26:428-38.

25. Ichikawa W. Prediction of clinical outcome of fluoropyrimidinebased chemotherapy for gastric cancer patients, in terms of the 5-fluorouracil metabolic pathway. Gastric Cancer. 2006;9:145-55.

26. Samuels Y, Wang Z, Bardelli A, Silliman N, Ptak J, Szabo S, et al. High frequency of mutations of the PIK3CA gene in human cancers. Science. 2004;304:554.

27. Kim S, Chun SY, Kwon YS, Nam KS. Crosstalk between Wnt signaling and Phorbol ester-mediated PKC signaling in MCF-7 human breast cancer cells. Biomed Pharmacother. 2016;77:114-9.

28. Civenni G, Holbro T, Hynes NE. Wnt 1 and Wnt5a induce cyclin D1 expression through ErbB1 transactivation in HC11 mammary epithelial cells. EMBO Rep. 2003;4:166-71.

29. Pukrop T, Klemm F, Hagemann T, et al. Wnt 5a signaling is critical for macrophage-induced invasion of breast cancer cell lines. Proc Natl Acad Sci USA. 2006;103:5454-9.

30. Yiu GK, Kaunisto A, Chin YR, Toker A. NFAT promotes carcinoma invasive migration through glypican-6. Biochem J. 2011;440:157-66.

31. Network CGA. Comprehensive molecular portraits of human breast tumours. Nature. 2012;490:61-70.

32. Deng L, Chen J, Zhong XR, Luo T, Wang YP, Huang HF, et al. Correlation between activation of $\mathrm{PI} 3 \mathrm{~K} / \mathrm{AKT} / \mathrm{mTOR}$ pathway and prognosis of breast cancer in Chinese women. PLoS ONE. 2015;10:e0120511.

33. Hanaki H, Yamamoto H, Sakane H, Matsumoto S, Ohdan H, Sato A, et al. An anti-Wnt5a antibody suppresses metastasis of gastric cancer cells in vivo by inhibiting receptor-mediated endocytosis. Mol Cancer Ther. 2012;11:298-307.

Publisher's Note Springer Nature remains neutral with regard to jurisdictional claims in published maps and institutional affiliations. 